\title{
miRNA Expression Profiling Regulates Epithelial Mesenchymal Transition in 125I Seed Irradiation of Gastric Carcinoma Cell
}

\section{Zhenhuan Ma}

The Affiliated Hospital of Yunnan University

\section{Zhen Li}

The Affiliated Hospital of Yunnan University

Lei Zou

affiliated hospital of Yunnan University

\section{Guojian Li}

The Affiliated Hospital of Yunnan University

\section{Yan Bao}

The Affiliated Hospital of Yunnan University

\section{Lingjuan Du}

The Affiliated Hospital of Yunnan University

\section{Yong Yang ( $\nabla$ yangyong_111@outlook.com )}

Kunming Medical University https://orcid.org/0000-0002-9115-1976

\section{Primary research}

Keywords: Gastric carcinoma, miR-1285-3p, epithelial mesenchymal transition, SMAD

Posted Date: November 8th, 2021

DOl: https://doi.org/10.21203/rs.3.rs-1002534/v1

License: (c) (i) This work is licensed under a Creative Commons Attribution 4.0 International License. Read Full License 


\section{Abstract}

Backgroud The aim of this study is to examine miRNA profiling and miR-1285-3p participates in 125I seed irradiation of gastric carcinoma cell via the regulation of epithelial mesenchymal transition (EMT).

Methods An in vitro l-125 seed irradiation model was established, followed by the small RNA-Sequencing to investigate the full spectrum of miRNAs that are response to $1-125$ seed implantation. Validation was performed with quantitative real time PCR (qRT-PCR). qRT-PCR was also employed to measure miR-1285$3 p$ and EMT-related mRNAs expression. Western blotting assay was performed to test the expression of EMT-related proteins. Luciferase reporter assay was conducted to confirm the direct targeting of SMAD2/3 and SMAD4 by miR-1285-3p.

Results A total of 1034 miRNAs were initially detected. Of these, 11 miRNAs were significantly differentially expressed between $1-125$ seed irradiation and control groups. Six miRNAs (hsa-miR-127-3p, hsa-miR-1285-3p, hsa-miR-296-5p, hsa-miR-421, hsa-miR-495-3p, and hsa-miR-548am-3p) were up regulation and five miRNAs (hsa-miR-17-5p, hsa-miR-193b-5p, hsa-miR-23b-5p, hsa-miR-483-5p, hsa-miR92a-1-5p) were down regulation between $1-125$ seed irradiation treatment and control groups. EMT is involved in gastric cancer cells treatment with $\mathrm{I}-125$ seed implantation, and downregulation of miR-1285$3 p$ can repress EMT through its targeting of SMAD pathway, which make miR-1285-3p a novel target of I125 therapeutic intervention for human gastric cancer.

Conclusions This study revealed that miR-1285-3p inhibited EMT by targeting SMAD pathway in 125I seed irradiation of gastric carcinoma.

\section{Background}

Gastric carcinoma (GC) is one of the most aggressive types of tumors [1] and the second leading cause of cancer-associated mortality as the gastrointestinal infectious disease in eastern Asia [2,3]. Various therapeutic strategies are currently available for GC patients, including surgery, chemotherapy, and radiotherapy. For early-stage gastric cancer, surgical resection remains a standard therapeutic approach. Besides surgical resection, endoscopic submucosal dissection has also been served as a common treatment for early-stage IA gastric cancer [4, 5]. Currently, surgery, chemotherapy and radiotherapy are therapeutic strategies for GC patients. In the USA and Europe, postoperative chemoradiotherapy could be an effective treatment for GC patients for controlling tumor progression in advanced gastric cancer [6-8]. Radioactive iodine-125 (I-125) seed implantation could be an effective and safer therapeutic strategy for advanced or unresectable gastric cancer [9]. Due to the high precision and fewer complications, I-125 seed implantation has been used in treatment of advanced gastric cancer [10]. In our previous study, we found that $\mathrm{I}-125$ seed irradiation induces up-regulation of the genes associated with apoptosis and cell cycle arrest and inhibits growth of gastric cancer xenografts [11]. Although several molecular mechanisms of I-125 seed implantation have been introduced, the inhibitory effect of I-125 remains not 
clearly[12-14]. Therefore, it is crucial to understand the molecular mechanism and it will be beneficial to achieve optimal outcomes of the I-125 seed implantation.

To investigate the complexity of the cellular response to $1-125$ seed implantation, microRNAs (miRNAs or miRs) were considered as the potential molecules involved in the mechanism of 1-125 implantation of GC. miRNAs are short non-coding RNAs given their broad effect on post-transcription, translational repression $[15,16]$. Over the past decades, miRNAs play a key role in a variety of cancer types [17-20]. More recently, using small RNA sequencing (RNA-Seq) technique could isolate and sequence small RNA species, such as miRNAs with unprecedented sensitivity and dynamic range. In the present study, we established an I125 seed irradiation model in vitro, followed by the small RNA-Seq to investigate the full spectrum of miRNAs that are response to $\mathrm{I}-125$ seed implantation.

\section{Methods}

\section{Cells and cell culture}

The gastric cancer cell line, BGC-823 was provided by the Cell Bank of the Chinese Academy of Sciences (Shanghai, China). Cells were cultured in RPMI-1640 medium supplemented with $10 \%(\mathrm{v} / \mathrm{v})$ fetal bovine serum (FBS, Gibco, Rockville, MD, USA) and $1 \%$ antibiotics at $37^{\circ} \mathrm{C}$ in a humidified incubator under $5 \%$ CO2 condition.

\section{I-125 seed irradiation model in vitro}

Type-6711 I-125 seeds were kindly provided by Ningbo Jun-An Pharmaceutical Technology Co. Ltd. (Ningbo, Zhejiang, China). I-125 seed irradiation model was performed according to a previous report[10]. Briefly, the seeds were $0.8 \mathrm{~mm}$ in diameter, $4.5 \mathrm{~mm}$ long, with a surface activity of $15.1-16.8 \mathrm{MBq}$, a halflife of $59.6 \mathrm{~d}$, and average energy of $27.4-35.5 \mathrm{Kev}$. Liquid paraffin was laid on the bottom of a 6-cm diameter cell culture dish. Eight I-125 seeds were evenly embedded within recesses $(4.5 \mathrm{~mm} \times 0.8 \mathrm{~mm})$ around a 35-mm diameter circumference, with one $\mathrm{l}-125$ seed placed in the center of the 6-cm dish, in order to obtain a relatively homogeneous dose distribution at the surface of the cell culture dish. A 6-cm culture dish was placed on the in-house $1-125$ irradiation model during the experiment. $1 \times 10^{5}$ cells of BGC-823 cell line were seeded into the 6 -cm culture dish and incubated for $96 \mathrm{~h}$ under constant cell culture conditions. All the culture dishes were rotated clockwise at specific time points to ensure even irradiation of the cells. The cells were divided into two different groups, I-125 seed irradiated group and control group (with no l-125 treatment).

\section{MicroRNA profling by small RNA-sequencing}

Total RNA was isolated using the miRNeasy mini kit (Qiagen, Shanghai, China), according to the manufacturer's instructions. The quantity and quality of RNA were assessed using the NanoDrop ND1000 instrument (Nanodrop Technologies, Wilmington, USA) and the Bioanalyser 2100 system (Agilent Technologies, CA, USA) using the Agilent RNA 6000 Nano and small RNA kits (Agilent Technologies), respectively, according to the manufacturer's instructions. The libraries for small RNA-sequencing were 
prepared using the TruSeq small RNA library kit from Illumina with 50ng of total RNA. The reverse transcription and PCR amplification (with bar-coded primers) were used to ligate RNA and 3' and 5' RNA adapters, according to the manufacturer's use. The Pippin Prep System was used to separate the PCR products into $147 \mathrm{nt}$ and $157 \mathrm{nt}$ fractions. High throughput sequencing service was provided by CloudSeq Biotech (Shanghai, China). The libraries were denatured as single-stranded DNA molecules, captured on Illumina flow cells, amplified in situ as clusters and finally sequenced for 50 cycles on Illumina HiSeq sequencer following the manufacturer's instructions. Raw data were generated after sequencing, image analysis, base calling, and quality filtering on Illumina sequencer. Firstly, Q30 was used to perform quality control. The adaptor sequences were trimmed and the adaptor-trimmed-reads ( $>=15 \mathrm{nt}$ ) were left by Cutadapt software (v1.9.3). Then, trimmed reads from all samples were pooled, and miRDeep2 software (v2.0.0.5) was used to predict novel miRNAs. The trimmed reads were aligned to the merged human premiRNA databases (known pre-miRNA from miRBase plus the newly predicted pre-miRNAs) using Novoalign software (v3.02.12) with at most one mismatch. The numbers of mature miRNA mapped tags were defined as the raw expression levels of that miRNA. The read counts were normalized by TPM (tag counts per million aligned miRNAs) approach. Differentially expressed miRNA between two groups were filtered through Fold change and $P$-value using the two tailed, homoscedastic $t$-test. miRNA targets were performed by popular miRNA target prediction softwares, miRNA-targets networks were plotted by cytoscape software (v2.8.0), and the GO and KEGG pathway analysis were performed based on the top 10 differentially expressed miRNA target genes.

\section{RNA isolation, cDNA synthesis and quantitative RT-PCR analysis}

For validation of the results of microRNA profling by small RNA-sequencing, quantitative RT-PCR was performed. Total RNA was extracted from cell cultures using TRIzol reagent (Invitrogen/Thermo Fisher Scientific, Inc., Shanghai, China), according to the manufacturer's instructions. Total RNA concentration and integrity were examined using an Agilent Bioanalyzer (Agilent Technologies, Inc., Santa Clara, CA, USA). Subsequently, $500 \mathrm{ng}$ of total mRNA per sample was reverse transcribed into cDNA using the High capacity cDNA Reverse Transcription kit (Applied Biosystems/Thermo Fisher Scientific, Inc.). For detection of miRNA expression, miDETECT A Track ${ }^{\text {TM }}$ qRT-PCR Kit (RiboBio Co., Ltd, Guangzhou, China) was used and RNU6 small nuclear RNA was used to normalize miRNA expression levels. For detection of mRNA expression, a standard TaqMan PCR kit procedure on an ABI-7500 system (Applied

Biosystems/Thermo Fisher Scientific, Inc.) according to the manufacturer's instructions. The mRNA levels were normalized to endogenous $\beta$-actin (Applied Biosystems). Primers and probes were shown in Table 1. Relative fold expression and changes were calculated using the 2- $\Delta \Delta \mathrm{Ct}$ method [21]. 
Table 1

Differentially expressed microRNAs identified by next generation sequencing

miRNA Information

\begin{tabular}{lllllll}
$\begin{array}{l}\text { mature- } \\
\text { miRNA }\end{array}$ & $\begin{array}{l}\text { pre- } \\
\text { miRNA }\end{array}$ & mature-sequence & $\begin{array}{l}\text { Fold } \\
\text { Change }\end{array}$ & $\begin{array}{l}\text { P- } \\
\text { value }\end{array}$ & FDR & Regulation \\
$\begin{array}{l}\text { hsa-miR- } \\
17-5 p\end{array}$ & $\begin{array}{l}\text { hsa-mir- } \\
17\end{array}$ & CAAAGUGCUUACAGUGCAGGUAG & 2.074 & 0.029 & 0.630 & down \\
$\begin{array}{l}\text { hsa-miR- } \\
\text { 193b-5p }\end{array}$ & $\begin{array}{l}\text { hsa-mir- } \\
193 b\end{array}$ & CGGGGUUUUGAGGGCGAGAUGA & 7.000 & 0.049 & 0.630 & down \\
$\begin{array}{l}\text { hsa-miR- } \\
\text { 23b-5p }\end{array}$ & $\begin{array}{l}\text { hsa-mir- } \\
23 b\end{array}$ & UGGGUUCCUGGCAUGCUGAUUU & 1.750 & 0.000 & 0.000 & down \\
$\begin{array}{l}\text { hsa-miR- } \\
483-5 p\end{array}$ & $\begin{array}{l}\text { hsa-mir- } \\
483\end{array}$ & AAGACGGGAGGAAAGAAGGGAG & 3.569 & 0.033 & 0.630 & down \\
$\begin{array}{l}\text { hsa-miR- } \\
92 a-1-5 p\end{array}$ & $\begin{array}{l}\text { hsa-mir- } \\
92 a-1\end{array}$ & AGGUUGGGAUCGGUUGCAAUGCU & 5.600 & 0.007 & 0.630 & down \\
$\begin{array}{l}\text { hsa-miR- } \\
127-3 p\end{array}$ & $\begin{array}{l}\text { hsa-mir- } \\
127\end{array}$ & UCGGAUCCGUCUGAGCUUGGCU & -2.033 & 0.036 & 0.630 & up \\
$\begin{array}{l}\text { hsa-miR- } \\
1285-3 p\end{array}$ & $\begin{array}{l}\text { hsa-mir- } \\
1285-1\end{array}$ & UCUGGGCAACAAAGUGAGACCU & -2.000 & 0.000 & 0.000 & up \\
$\begin{array}{l}\text { hsa-miR- } \\
1285-3 p\end{array}$ & $\begin{array}{l}\text { hsa-mir- } \\
1285-2\end{array}$ & UCUGGGCAACAAAGUGAGACCU & -2.000 & 0.000 & 0.000 & up \\
$\begin{array}{l}\text { hsa-miR- } \\
296-5 p\end{array}$ & $\begin{array}{l}\text { hsa-mir- } \\
296\end{array}$ & AGGGCCCCCCCUCAAUCCUGU & -2.667 & 0.038 & 0.630 & up \\
$\begin{array}{l}\text { hsa-miR- } \\
421\end{array}$ & $\begin{array}{l}\text { hsa-mir- } \\
421\end{array}$ & AUCAACAGACAUUAAUUGGGCGC & -1.750 & 0.000 & 0.000 & up \\
$\begin{array}{l}\text { hsa-miR- } \\
495-3 p\end{array}$ & $\begin{array}{l}\text { hsa-mir- } \\
495\end{array}$ & AAACAAACAUGGUGCACUUCUU & -7.714 & 0.034 & 0.630 & up \\
$\begin{array}{l}\text { hsa-miR- } \\
548 a m- \\
3 p\end{array}$ & $\begin{array}{l}\text { hsa-mir- } \\
\text { h48am }\end{array}$ & CAAAAACUGCAGUUACUUUUGU & -3.333 & 0.020 & 0.630 & up \\
\hline
\end{tabular}

\section{Luciferase activity assay}

Human SMAD6-3' UTR containing the putative target site for miR-1285-3p was amplified by PCR and inserted into the pmiR-RB-REPORT (RiboBio Co., Ltd, Guangzhou, China), as well as the mutation. The cells were transiently transfected with the wild type or mutant reporter plasmids, miR-1285-3p mimic, and NC mimic using Lipofectamine 3000 (Thermo Fisher Scientific, Inc. Shanghai, China). Luciferase activity was measured 48 hours after transfection using the dual-luciferase assay system (Promega Co., Ltd, Bejing, China). Three independent experiments were performed in triplicate.

\section{Western blot analysis}


Total protein was extracted from the cells RIPA lysis buffer. Protein concentrations were measured using a BCA Protein Assay Kit (Beyotime Biotechnology, Shanghai, China). $50 \mu \mathrm{g}$ of extracted protein were assayed with SDS-polyacrylamide gels (containing a $12 \%$ separating gel and a $5 \%$ stacking gel). The protein was subsequently transferred onto a polyvinyl difluoride (PVDF) membrane (Roche Applied Science, Shanghai, China). The membranes were stripped and probed with the following primary antibodies: anti-GAPDH (Kangchen Biotechnology, Shanghai, China), anti-SMAD6 (Abcam, Shanghai, China), anti-E-Cadherin (BD Transduction Laboratories, Beijing, China), anti-Vimentin (Abcam, Shanghai, China), and anti-alpha-SMA (Abcam, Shanghai, China). Quantification of Western blots was processed with ImageJ software.

\section{Statistical analysis}

Statistical analysis was performed using PRISM version 5.0 software (GraphPad, San Diego, CA, USA). qPCR data are presented as the mean \pm standard error of the mean (SEM). Statistical significance was determined using an unpaired $t$-test and the One way analysis of variance (ANOVA), followed by Tukey post hoc. A $P \leq 0.05$ was considered statistically significant. Statistical analysis of sequencing and bioinformatics results are discussed in the relevant sections.

\section{Results}

\section{Results of microRNA Expression Profiling by small RNA- sequencing}

To study the full spectrum of miRNAs involved in I-125 seed irradiation, gastric cancer cell line, BGC-823 was assayed after treatment with or without (no seeds as a control) irradiation. Small RNA- sequencing was performed and a total of 1034 miRNAs were initially detected. Of these, 11 miRNAs were significantly differentially expressed between $1-125$ seed irradiation and control groups. Six miRNAs (hsa-miR-127-3p, hsa-miR-1285-3p, hsa-miR-296-5p, hsa-miR-421, hsa-miR-495-3p, and hsa-miR-548am-3p) were up regulation and five miRNAs (hsa-miR-17-5p, hsa-miR-193b-5p, hsa-miR-23b-5p, hsa-miR-483-5p, hsa-miR92a-1-5p) were down regulation between $1-125$ seed irradiation treatment and control groups (Table 1).

qRT-PCR was then used to validate the results of small RNA-Sequencing. The qRT-PCR results demonstrated an increase in the expression of hsa-miR-127-3p (5.50 \pm 0.24 -fold), hsa-miR-1285-3p (3.45 \pm 0.22 -fold), hsa-miR-296-5p (4.63 \pm 0.18 -fold), hsa-miR-421 (5.70 \pm 0.25 -fold), hsa-miR-495-3p (2.39 \pm 0.07 -fold), and hsa-miR-548am-3p (3.52 \pm 0.08 -fold) in the I- 125 treatment group compared to I-125 no treatment group, whereas the expression of hsa-miR-17-5p (4.49 $\pm 0.09-f o l d)$, hsa-miR-193b-5p (2.70 \pm 0.16 -fold), hsa-miR-23b-5p (5.60 \pm 0.22 -fold), hsa-miR-483-5p (3.71 \pm 0.20 -fold), hsa-miR-92a-1-5p $(4.43 \pm 0.28$-fold) decreased, respectively (Figure 1$)$. These data were consistent with the small RNASequencing results.

Then, we performed a computational approach to predict the miRNA target genes. The prediction of miRNA target genes was performed with the following three different miRNA target prediction algorithms: 
PicTar, miRanda v5 and TargetScan v5.1. Each algorithm has a definite rate of both false positive and false negative predictions. Based on these database searches, the genes with target sites for all of three co-expressed miRNAs were identified as a potential cooperative target gene set. Then, these results were integrated into the gene network analysis using the software Medusa (Figure 2).

\section{Weakened EMT and SMAD pathway after GC cells treated with I-125}

In I-125 treated GC cells, the expression of EMT markers and the key factors of SMAD pathway were significantly different from no I-125 treatment. The expression of E-cadherin was significantly upregulated at the protein levels compared with that in the normal controls. We also found the expression of Vimentin, alpha-SMA, SMAD2/3, and SMAD4 were more reduced in the I-125 treated cells than that in the normal controls (Figure 3).

\section{miR-1285-3p regulated EMT of I-125 treated GC cells by targeting SMAD pathway}

To understand the mechanisms by which miR-1285-3p regulated EMT of I-125, several miRNA target prediction algorithms, including Microcosm (http://www.ebi.ac. uk/enrightsrv/microcosm/htdocs/targets/v5/), starBase (http://starbase.sysu.edu.cn/), Pictar (http://pictar.mdcberlin.de/) and TargetScan (http://www.targetscan.org/) were used to identify the potential target genes of miR-1285-3p. A total of 9 target genes were predicted by all four prediction algorithms used in the present study (Table 2). Among these genes, SMAD2/3, SMAD4 was also demonstrated to be targeted by miR-1285-3p from experimental data in the DIANA TarBase v7.0 database (http://diana.imis.athenainnovation.gr/DianaTools/index.php?r=tarbase/), and therefore we focused on SMAD pathway. To determine whether SMAD pathway was a genuine target of miR-1285-3p, a set of functional experiments was performed. First, miR-1285-3p mimic was transfected into BGC-823, and SMAD2/3, SMAD4 expression was determined at both the mRNA and protein levels. The expression of SMAD2/3, SMAD4 was downregulated following miR-1285-3p mimic overexpression (Figure. 4A and 4B). To further confirm that miR-1285-3p could directly bind to SMAD2 or SMAD3 or SMAD4 and inhibit their expression, a luciferase reporter plasmid was generated with wild-type or mutant sequence of the SMAD2 or SMAD3 or SMAD4 predicted mRNA target fragment. The reporter constructs were co-transfected with miR-1285-3p mimics or negative control oligonucleotides into cells for $48 \mathrm{~h}$, and then luciferase activity was measured in the transfected cells. The results confirmed that the reporter construct with wild-type targeting sequence of SMAD2 or SMAD3 or SMAD4 mRNA caused a significant decrease in luciferase activity in cells transfected with miR-1285-3p, whereas the reporter construct with mutant sequence of SMAD2 or SMAD3 or SMAD4 produced no change in luciferase activity. These results suggested that miR-1285-3p could bind to SMAD2 or SMAD3 or SMAD4 directly and inhibit their expression (Figure. 4C). 
Table 2

List of the 9 predicted target genes for miR-1285-3p.

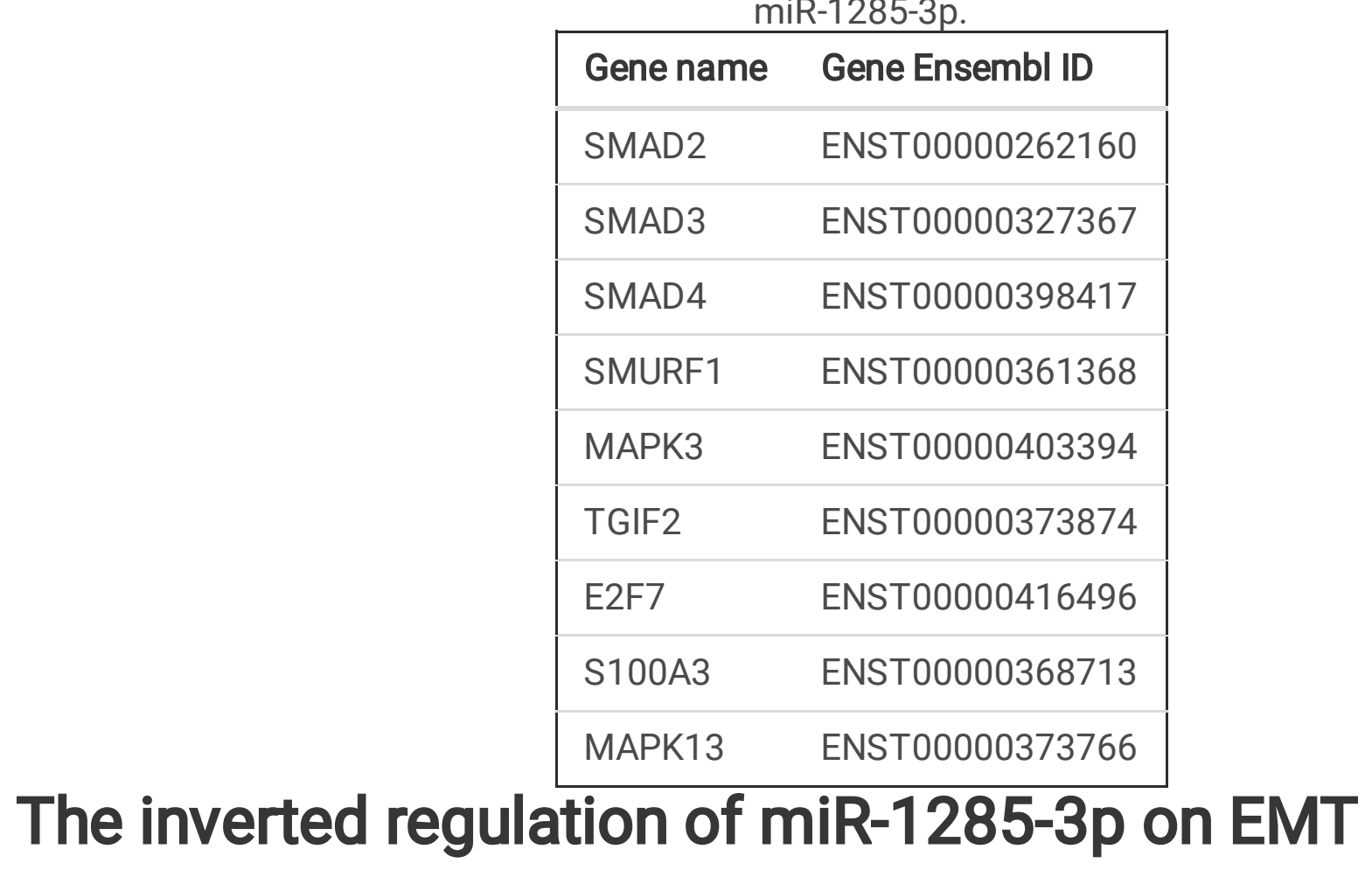

To further investigate the role of miR-1285-3p in repressing EMT in the I-125 seed irradiation model in vitro, protein levels of vimentin and alpha-SMA were detected. The expression of both vimentin and alpha-SMA proteins was downregulated after the BGC-823 cells were treated with the miR-1285-3p inhibitor (inhibitor NC as control, $\mathrm{P}<0.05$, Figure. 5A). Moreover, the BGC-823 cells were treated with transforming growth factor-beta2 (TGF-beta2) and a miR-1285-3p inhibitor. In the TGF-beta2 group, alpha-SMA and vimentin were high expressed relative to the group without TGF-beta2 treatment, suggesting an increased EMT level induced by TGF-beta2. By contrast, downregulation of miR-1285-3p decreased the alpha-SMA expression and vimentin expression (Figure. 5B). MiR-1285-3p could downregulate the levels of EMT induced by TGF-beta2 in the I-125 seed irradiation model.

\section{Discussion}

Understanding of the functions of miRNAs on EMT in radiotherapy could provide the means for discovering novel therapies for gastric carcinoma. In the present study, miR-1285-3p was observed to be upregulated in $\mathrm{l}-125$ seed irradiation model in vitro, and miR-1285-3p targeted SMAD pathway and inhibited EMT when the GC cells were treated with $1-125$ seed irradiation. We used small RNA-sequencing to study the full spectrum of miRNAs that are expressed in $1-125$ seed irradiation model in vitro. We demonstrate, for the first time, that $1-125$ seed irradiation is characterized by unique miRNA expression signatures and use small RNA sequencing profles of GC cells to identify novel putative oncogenic or tumor suppressive miRNAs in the context of human gastric carcinoma. 
According to several reports, continuous low-dose-rate irradiation by $1-125$ seeds is critical in apoptosis induction and cell-cycle arrest [22,23]. We have also found $125 \mathrm{I}$ seed irradiation induces up-regulation of the genes associated with apoptosis and cell cycle arrest and inhibits growth of gastric cancer xenografts [11]. That study revealed that $125 \mathrm{I}$ seed irradiation could significantly induce the up-regulation of apoptosis- and cell cycle-related genes in human gastric cancer xenografts. And some of the upregulation might be attributed to 125 l-irradiation induced demethylation in gene promoter regions.

As we know, the primary tumour is generally well operable, as a result gastric cancer related mortality is caused by distant metastases, not by the primary tumour. It is therefore important to gain insight into the process of gastric cancer dissemination. One of the most important steps is the acquisition of motility and invasive properties by cancer cells, associated with epithelial-mesenchymal transition (EMT) [24]. Epithelial plasticity is regulated by various pathways, the transforming growth factor (TGF)- $\beta$ pathway being a well-studied inducer of EMT and gastric cancer invasion and metastasis [25].

Several miRNAs have been implicated as modulators of EMT in gastric cancer. Zhou, $X$ et al. found that miR-200c inhibits TGF-beta-induced-EMT to restore trastuzumab sensitivity by targeting ZEB1 and ZEB2 in gastric cancer [26]. Huang, $\mathrm{J}$ et al. reported that miR-302b inhibits tumorigenesis by targeting EphA2 via Wnt/ beta-catenin/EMT signaling cascade [27]. miR-616-3p promotes angiogenesis and EMT in gastric cancer via the PTEN/AKT/mTOR pathway [28]. miR-630 inhibits EMT by regulating Wnt/betacatenin pathway in gastric cancer cells [29].

Recently, upregulation of hsa-miR-1285 was demonstrated in bronchoalveolar lavage fluid samples from patients with lung cancer and downregulation in plasma level of stage-l lung cancer patients. miR-1285$5 p$ functions as a tumor promoter in the development of non-small-cell lung carcinoma by targeting Smad4 and $\mathrm{CDH} 1[30]$. miRNA-1285 inhibits malignant biological behaviors of human pancreatic cancer cells by negative regulation of YAP1 [31]. In our present study, we found that I-125 seed implantation induced the expression of miR-1285-3p and inhibit the activation of SMAD pathway, which is the critical pathway in EMT. We will continue our study on the potential therapeutic role of miR-1285-3p to increase the effect of I-125 seed implantation therapy.

\section{Conclusions}

In conclusion, we identified that EMT is involved in gastric cancer cells treatment with $1-125$ seed implantation, and downregulation of miR-1285-3p can repress EMT through its targeting of SMAD pathway, which make miR-1285-3p a novel target of $\mathrm{I}-125$ therapeutic intervention for human gastric cancer.

\section{Abbreviations}

GC

Gastric carcinoma 
EMT

Epithelial mesenchymal transition

miR

microRNA

\section{Declarations}

\section{Authors' contributions}

ZM, ZL and YY participated in the conception and design of the study. ZM, ZL and LZ contributed the cell culture and treatment for the study. GL, YB and LD performed the data collection and analysis. ZM, ZL and $Y Y$ contributed the critical revision of the manuscript for important intellectual content. ZM and $Y Y$ interpreted the data and produced the draft of the manuscript. All authors read and approved the final manuscript.

\section{Author details}

1 The third Department of General Surgery, Affiliated Hospital of Yunnan University, 176 Youth Road, Kunming, 650021, Yunnan Province, P.R. China

2 The Third Department of General Surgery, Fourth Affiliated Hospital of Kunming Medical University, Kunming, 650021, Yunnan Province, P.R. China

3 Department of Organ Transplant, First Affiliated Hospital of Kunming Medical University, Kunming, 650032, Yunnan Province, P.R. China.

\section{Acknowledgements}

The authors want to thank Meifang Dai (Shanghai Yunxu Biotechnology Co., Ltd) for the valuable help.

\section{Competing interests}

The authors declare that they have no competing interests.

\section{Availability of data and materials}

The datasets used and/or analyzed during the current study are available from the corresponding author on reasonable request.

\section{Consent for publication}

Not applicable.

\section{Ethics approval and consent to participate}


This study was approved by the Review Board of the Affiliated Hospital of Yunnan University under the supervision of the Ethics Committee.

\section{Funding}

This study was supported by (1) the Research Projects of Medical Institutions in Yunnan Province (2017NS137; 2018NS0008; 2018NS0009); (2) the medical discipline reserve talents of Yunnan province $\mathrm{H}-2017037$.

\section{Publisher's Note}

Springer Nature remains neutral with regard to jurisdictional claims in published maps and institutional afliations.

\section{References}

1. Herszenyi L, Tulassay Z: Epidemiology of gastrointestinal and liver tumors. European review for medical and pharmacological sciences 2010, 14(4):249-258.

2. Roder DM: The epidemiology of gastric cancer. Gastric cancer : official journal of the International Gastric Cancer Association and the Japanese Gastric Cancer Association 2002, 5 Suppl 1:5-11.

3. Terry MB, Gaudet MM, Gammon MD: The epidemiology of gastric cancer. Seminars in radiation oncology 2002, 12(2):111-127.

4. Hoteya S, Yahagi N, lizuka T, Kikuchi D, Kawano K, Noguchi T, Mizuno H, Hashimoto M: [Endoscopic resection for early gastric cancers by EMR/ESD]. Gan to kagaku ryoho Cancer \& chemotherapy 2007, 34(1):16-20.

5. Hoteya S, Yamashita S, Kikuchi D, Nakamura M, Fujimoto A, Matsui A, Nishida N, Mitani T, Kuroki Y, lizuka $T$ et al: Endoscopic submucosal dissection for submucosal invasive gastric cancer and curability criteria. Digestive endoscopy : official journal of the Japan Gastroenterological Endoscopy Society 2011, 23(1):30-36.

6. Dong HM, Wang Q, Wang WL, Wang G, Li XK, Li GD, Chen J: A clinical analysis of systemic chemotherapy combined with radiotherapy for advanced gastric cancer. Medicine 2018, 97(23):e10786.

7. Yuan SQ, Wu WJ, Qiu MZ, Wang ZX, Yang LP, Jin Y, Yun JP, Gao YH, Li YH, Zhou ZW et al: Development and Validation of a Nomogram to Predict the Benefit of Adjuvant Radiotherapy for Patients with Resected Gastric Cancer. Journal of Cancer 2017, 8(17):3498-3505.

8. Stumpf PK, Amini A, Jones BL, Koshy M, Sher DJ, Lieu CH, Schefter TE, Goodman KA, Rusthoven CG: Adjuvant radiotherapy improves overall survival in patients with resected gastric adenocarcinoma: $\mathrm{A}$ National Cancer Data Base analysis. Cancer 2017, 123(17):3402-3409.

9. Ju Z, Wang Z, Wang L, Li J, Wu Z, Li X, Wang F, Wang R: Experimental study on radiation damage of(125)I seeds implanted in canine gastric wall tissue. Journal of cancer research and therapeutics 
2020, 16(2):203-208.

10. Takabayashi K, Kashiwagi K, Kawata T, Sato T, Matsuoka K, Hisamatsu T, Takaishi H, Hibi T, Ogata H, Yahagi $\mathrm{N}$ et al: Continuous low-dose irradiation by $\mathrm{l}-\mathbf{1 2 5}$ seeds induces apoptosis of gastric cancer cells regardless of histological origin. Cancer biology \& therapy 2014, 15(1):81-88.

11. Ma ZH, Yang Y, Zou L, Luo KY: 125I seed irradiation induces up-regulation of the genes associated with apoptosis and cell cycle arrest and inhibits growth of gastric cancer xenografts. Journal of experimental \& clinical cancer research : CR 2012, 31:61.

12. Liu XH, Chen GG, Vlantis AC, Tse GM, van Hasselt CA: lodine induces apoptosis via regulating MAPKs-related p53, p21, and Bcl-xL in thyroid cancer cells. Molecular and cellular endocrinology 2010, 320(1-2):128-135.

13. Rosner $\mathrm{H}$, Torremante P, Moller W, Gartner R: Antiproliferative/cytotoxic activity of molecular iodine and iodolactones in various human carcinoma cell lines. No interfering with EGF-signaling, but evidence for apoptosis. Experimental and clinical endocrinology \& diabetes : official journal, German Society of Endocrinology [and] German Diabetes Association 2010, 118(7):410-419.

14. Yokoyama A, Tanaka Y, Yokoyama T, Mizukami T, Matsui T, Maruyama K, Omori T: p53 protein accumulation, iodine-unstained lesions, and alcohol dehydrogenase-1B and aldehyde dehydrogenase-2 genotypes in Japanese alcoholic men with esophageal dysplasia. Cancer letters 2011, 308(1):112-117.

15. Ha M, Kim VN: Regulation of microRNA biogenesis. Nature reviews Molecular cell biology 2014, 15(8):509-524.

16. Jonas $\mathrm{S}$, Izaurralde $\mathrm{E}$ : Towards a molecular understanding of microRNA-mediated gene silencing. Nature reviews Genetics 2015, 16(7):421-433.

17. Esquela-Kerscher A, Slack FJ: Oncomirs - microRNAs with a role in cancer. Nature reviews Cancer 2006, 6(4):259-269.

18. Wang $Y$, Taniguchi T: MicroRNAs and DNA damage response: implications for cancer therapy. Cell cycle 2013, 12(1):32-42.

19. Zhang B, Pan X, Cobb GP, Anderson TA: microRNAs as oncogenes and tumor suppressors. Developmental biology 2007, 302(1):1-12.

20. Yong W, Zhang K, Deng Y, Tang W, Tao R: miR-511-5p Suppresses Cell Migration, Invasion and Epithelial-Mesenchymal Transition Through Targeting PAK2 in Gastric Cancer. Biochem Genet 2021.

21. Livak KJ, Schmittgen TD: Analysis of relative gene expression data using real-time quantitative PCR and the 2(-Delta Delta C(T)) Method. Methods 2001, 25(4):402-408.

22. Wang J, Wang J, Liao A, Zhuang $H$, Zhao $Y$ : The direct biologic effects of radioactive 125 I seeds on pancreatic cancer cells PANC-1, at continuous low-dose rates. Cancer biotherapy \& radiopharmaceuticals 2009, 24(4):409-416.

23. Zhuang HQ, Wang JJ, Liao AY, Wang JD, Zhao Y: The biological effect of 125 I seed continuous low dose rate irradiation in CL187 cells. Journal of experimental \& clinical cancer research : CR 2009, 28:12. 
24. Ye X, Weinberg RA: Epithelial-Mesenchymal Plasticity: A Central Regulator of Cancer Progression. Trends in cell biology 2015, 25(11):675-686.

25. Cao QH, Liu F, Li CZ, Liu N, Shu M, Lin Y, Ding L, Xue L: Testes-specific protease 50 (TSP50) promotes invasion and metastasis by inducing EMT in gastric cancer. BMC cancer 2018, 18(1):94.

26. Zhou X, Men X, Zhao R, Han J, Fan Z, Wang Y, Lv Y, Zuo J, Zhao L, Sang M et al: miR-200c inhibits TGF-beta-induced-EMT to restore trastuzumab sensitivity by targeting ZEB1 and ZEB2 in gastric cancer. Cancer gene therapy 2018, 25(3-4):68-76.

27. Huang J, He Y, McLeod HL, Xie Y, Xiao D, Hu H, Chen P, Shen L, Zeng S, Yin X et al: miR-302b inhibits tumorigenesis by targeting EphA2 via Wnt/ beta-catenin/EMT signaling cascade in gastric cancer. BMC cancer $2017,17(1): 886$.

28. Wu ZH, Lin C, Liu CC, Jiang WW, Huang MZ, Liu X, Guo WJ: MiR-616-3p promotes angiogenesis and EMT in gastric cancer via the PTEN/AKT/mTOR pathway. Biochemical and biophysical research communications 2018, 501(4):1068-1073.

29. Li D, Tian B, Jin X: miR-630 inhibits epithelial-to-mesenchymal transition (EMT) by regulating Wnt/betacatenin pathway in gastric cancer cells. Oncology research 2018.

30. Zhou S, Zhang Z, Zheng P, Zhao W, Han N: MicroRNA-1285-5p influences the proliferation and metastasis of non-small-cell lung carcinoma cells via downregulating CDH1 and Smad4. Tumour biology : the journal of the International Society for Oncodevelopmental Biology and Medicine 2017, 39(6):1010428317705513.

31. Huang $H$, Xiong G, Shen $P$, Cao Z, Zheng L, Zhang T, Zhao Y: MicroRNA-1285 inhibits malignant biological behaviors of human pancreatic cancer cells by negative regulation of YAP1. Neoplasma 2017, 64(3):358-366.

\section{Figures}




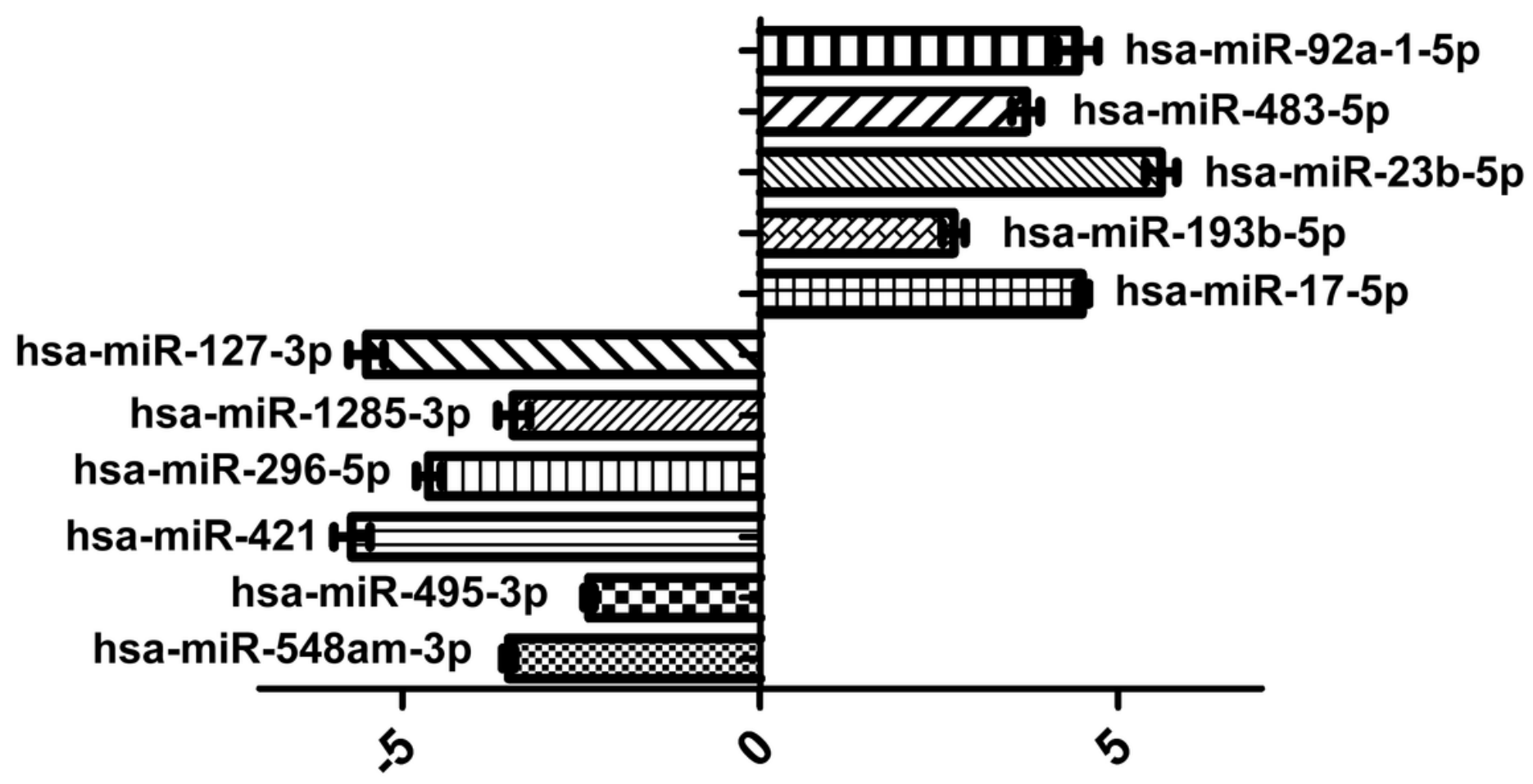

Fold change

Figure 1

Validation of selected microarray data by quantitative reverse transcription polymerase chain reaction. Relative expression levels of selected microRNAs (miRNAs) were determined by quantitative reverse transcription polymerase chain reaction (qRT-PCR). Values are mean \pm standard deviation (SD) and expressed relative to internal control (U6; $n=3$ for each group, ${ }^{*} P<0.05$, Student $t$ test). 
A
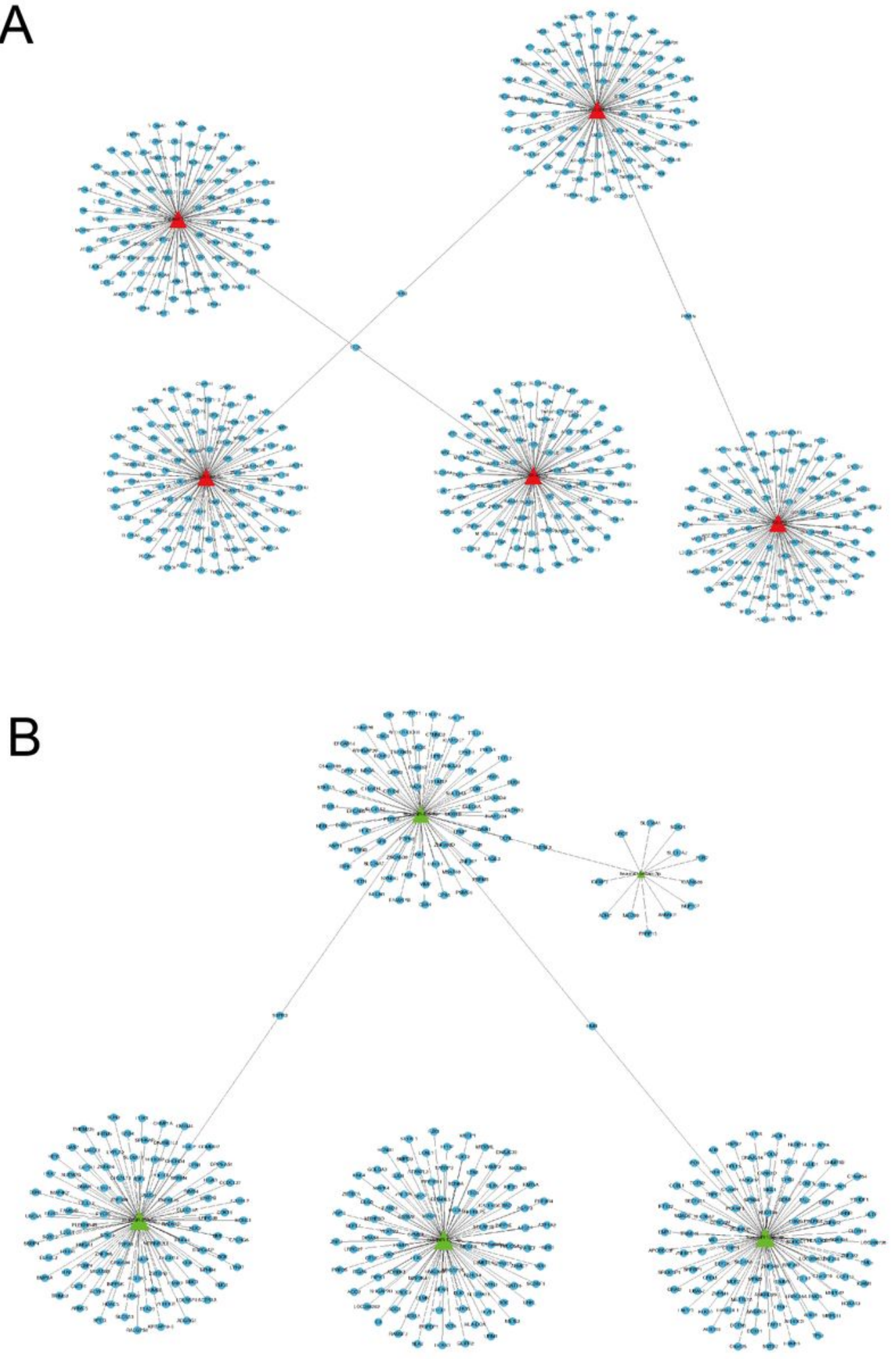

Figure 2

Results of gene network analysis. A computational approach was performed to predict the miRNA target genes with three different miRNA target prediction algorithms: PicTar, miRanda v5 and TargetScan v5.1. Each algorithm has a definite rate of both false positive and false negative predictions. Based on these database searches, the genes with target sites for all of three co-expressed miRNAs were identified as a 
potential cooperative target gene set. Then, these results were integrated into the gene network analysis using the software Medusa.
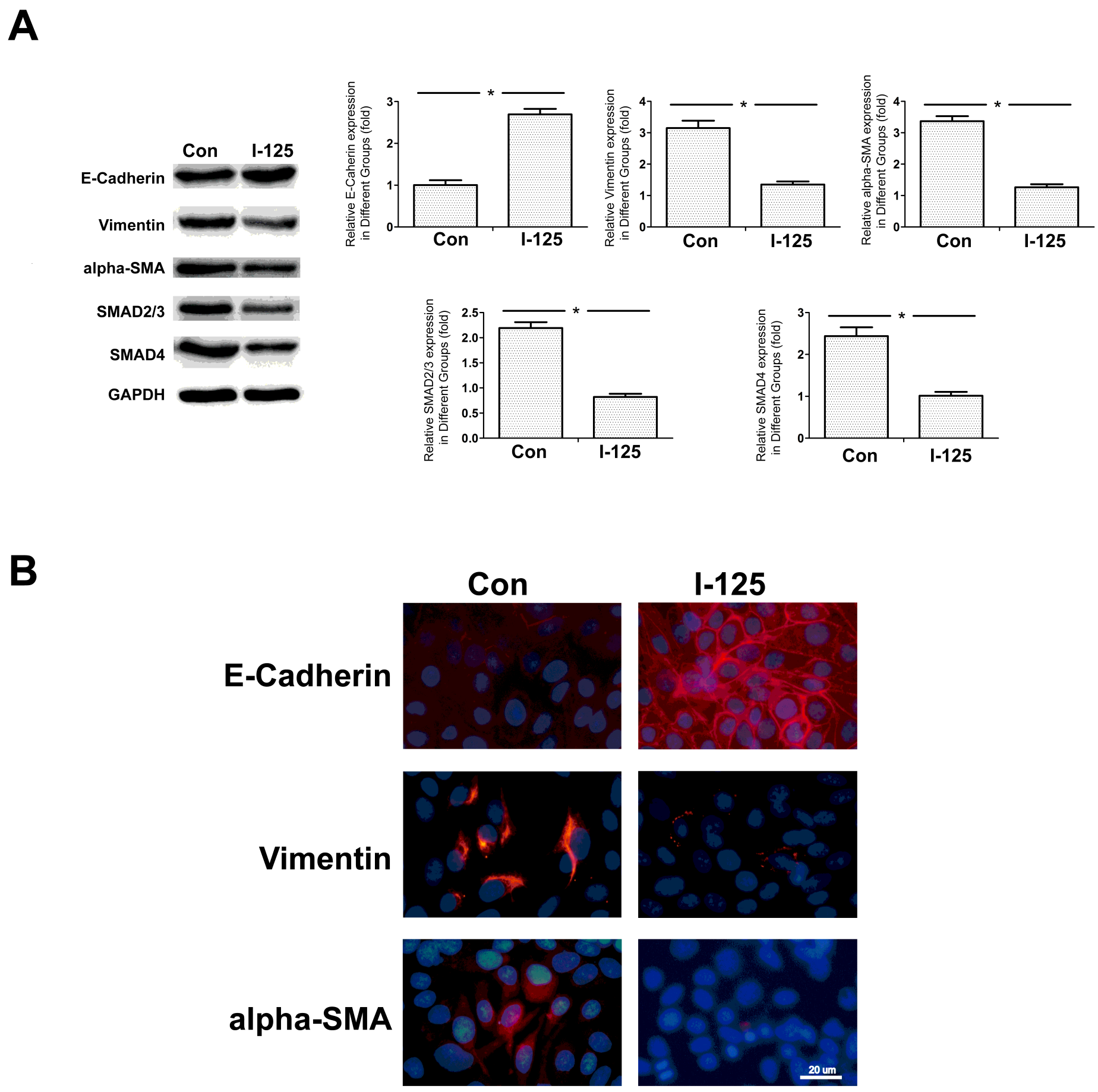

Figure 3

Expression of EMT markers in $1-125$ treated cells and normal controls. (A) Changes in protein expression as determined by Western blot. Expression of E-cadherin was higher in I-125 treated cells than in the normal control $(P<0.05, n=3)$. However, the expression of vimentin, alpha-SMA, SMAD2/3 and SMAD4 were lower in the $1-125$ treated cells than in the normal control $(P<0.05, n=3)$. (Left, data from the gels; Right, normalization to GAPDH). Significant differences between the $\mathrm{I}-125$ treated cells and normal 
control are indicated by an asterisk ( $\left.{ }^{*} P<0.05\right)$. (B) Results of the immunofluorescence analysis. $1-125$ treated cells demonstrated high E-cadherin expression and little or no expression of vimentin and alphaSMA. In contrast, E-cadherin was down-regulated, and vimentin and alpha-SMA were up-regulated in the normal control.

A
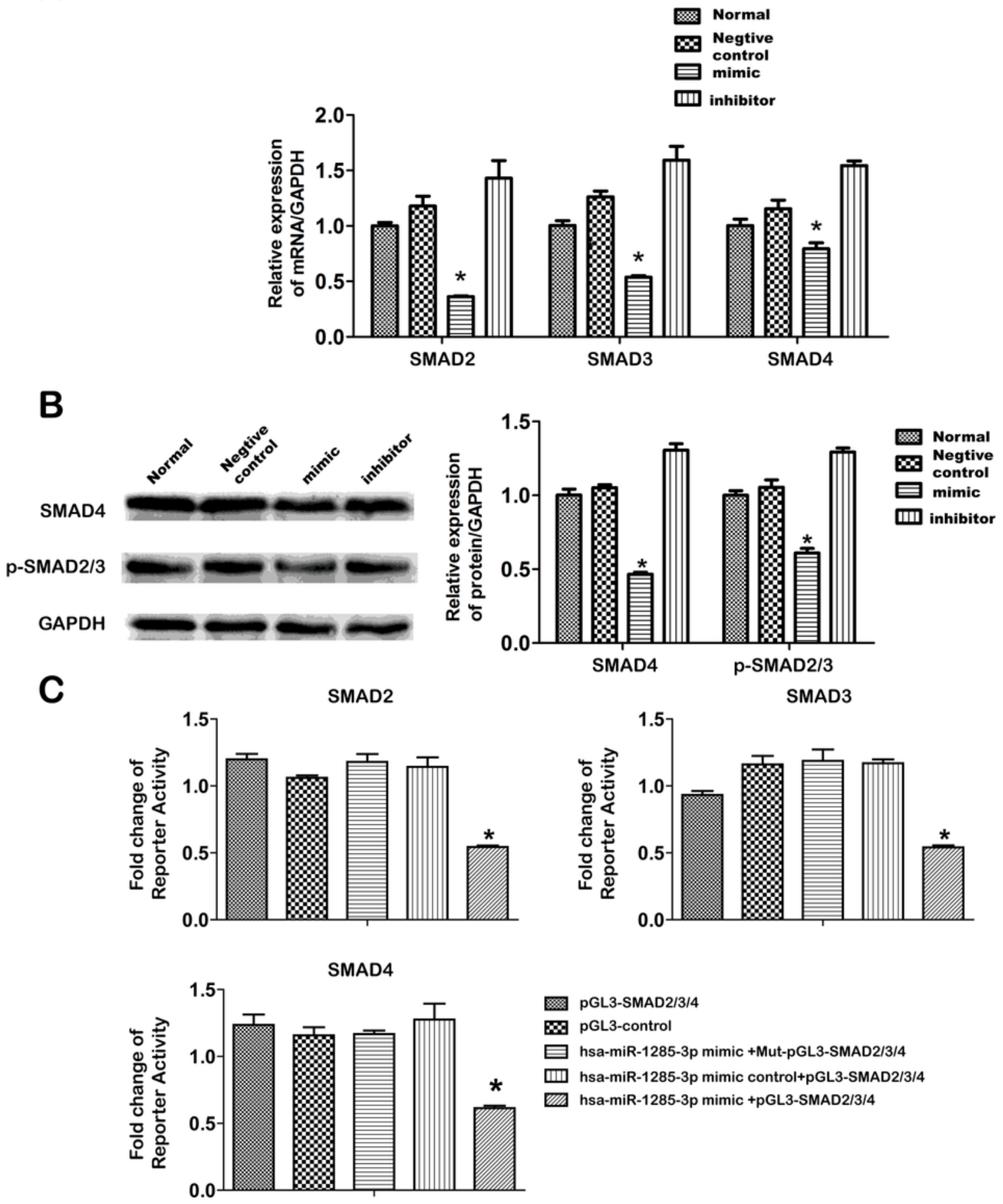

Figure 4 
miR-1285-3p directly targets SMAD pathway. (A) Effect of miR-1285-3p mimic overexpression on the endogenous SMAD2, SMAD3, and SMAD4 mRNA levels ( $n=3$ ). (B) Effect of miR-1285-3p mimic overexpression on the endogenous SMAD2, SMAD3, and SMAD4 protein levels $(n=3)$. (C) Luciferase reporter construct was made by cloning the human SMAD2 or SMAD3, or SMAD4 mRNA sequence into Reporter system. Wild-type or mutant SMAD2 or SMAD3, or SMAD4 mRNA fragments were also amplified and cloned into the luciferase reporter. BGC-823 cells were co-transfected with the reporter constructs bearing the wild type and mutant SMAD2 or SMAD3, or SMAD4 sequences, and with miR-1285-3p mimics or negative control oligonucleotides. After $48 \mathrm{~h}$, luciferase activity was measured and normalized to Renilla luciferase activity. Data are presented as the mean \pm standard error of the mean $\left({ }^{*} P<0.05\right)$. MUT, mutant.
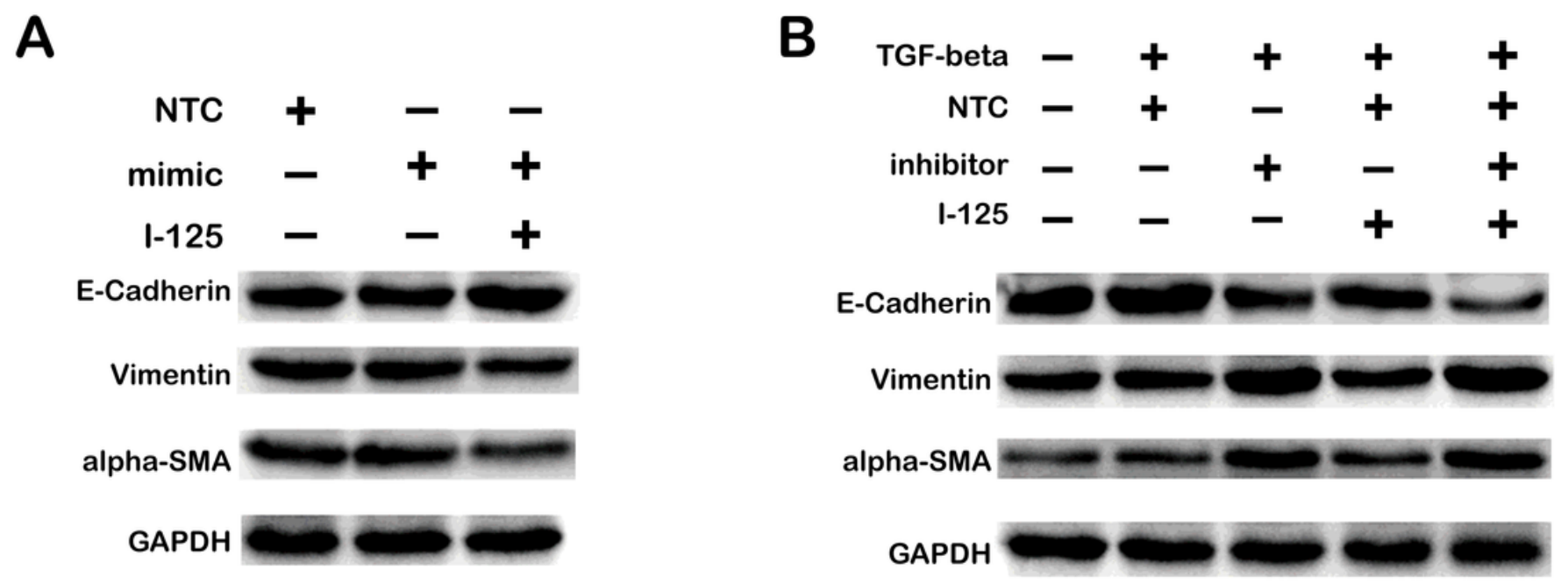

\section{Figure 5}

Upregulation of miR-1285-3p repressed EMT. (A) Protein expression levels of the EMT markers. The protein expression of vimentin and alpha-SMA was decreased when the BGC-823 cells were transfected with the miR-1285-3p mimic in $1-125$ treatment conditions ( ${ }^{*} \mathrm{P}<0.05$, mimic NC as control, $N=2$ ). (B) BGC-823 cells were treated with TGF-beta and a mimic of miR-1285-3p. In the presence of TGF-beta, EMT was induced with the increased protein levels of vimentin and alpha-SMA, as well as SMAD2 and SMAD3, and SMAD4 ( ${ }^{*}<<0.05, \mathrm{l}-125$ treatment without TFG-beta as control, $\mathrm{N}=2$ ), while the miR-1285-3p mimic repressed vimentin and alpha-SMA $(P<0.05$, mimic $N C$ as control, $N=2)$. NC, negative control 